Case

Report

\title{
Delayed Closure Technique Using the Ventral Hernia Repair Prosthesis for a Ruptured Abdominal Aortic Aneurysm: Report of a Case
}

Kazuki Hisatomi, MD, Takafumi Yamada, MD, and Daisuke Onohara, MD

\begin{abstract}
In spite of modern advances in medical care, the operative mortality of ruptured abdominal aortic aneurysm remains high at $40 \%-50 \%$. Multiple organ failure is one of the reasons for the high mortality rates. An acute increase in intra-abdominal pressure and abdominal compartment syndrome are common causes of multiple organ failure. It is important to prevent abdominal compartment syndrome to improve the outcome of ruptured abdominal aortic aneurysm. Delayed abdominal closure is effective in preventing abdominal compartment syndrome in patients with ruptured abdominal aortic aneurysm. We successfully achieved delayed abdominal closure using the ventral hernia repair prosthesis for a ruptured abdominal aortic aneurysm, in a straightforward and rapid manner. No infection was seen, secondary closure was readily performed, and wound healing was good. We conclude that our delayed closure technique is useful for the treatment of ruptured abdominal aortic aneurysm.
\end{abstract}

Keywords: delayed closure, abdominal compartment syndrome, ruptured abdominal aortic aneurysm

\section{Introduction}

In contrast and possible ongoing resuscitation to elective repair, the operative mortality of ruptured abdominal aortic aneurysm (rAAA) has not improved significantly in recent years, with mortality rates still ranging from about $40 \%-50 \% .^{1,2)}$ In these patients, multiple organ failure (MOF) in the acute postoperative period represents a significant cause of death., ${ }^{3,4)}$ Abdominal compartment syndrome (ACS) has been identified as an independent predictor of mortality in critically ill patients and is a common cause of MOF. ${ }^{4)}$ Patients who have survived a rAAA have typically

Department of Cardiovascular Surgery, Oita Prefectural Hospital, Oita, Japan

Received: March 7, 2012; Accepted: June 8, 2012

Corresponding author: Kazuki Hisatomi, MD. Department of Cardiovascular Surgery, Oita Prefectural Hospital, 476 Bunyo, Oita 870-8511, Japan

Email: hisatomi@oitakenbyo.jp

(C)2013 The Editorial Committee of Annals of Thoracic and Cardiovascular Surgery. All rights reserved. had a period of tissue ischemia prior to surgery or intra-operative debt. This ischemia leads to vasoconstriction, especially in the peripheral and splanchnic circulation. Postoperative resuscitation in the setting of reperfusion injury leads to a systemic inflammatory response and secondary visceral edema. ${ }^{5,6)}$ In turn, visceral edema may lead to ACS. Additionally, patients suffering from rAAA are at risk of ACS because they often have a large retroperitoneal hematoma that expands into the abdominal domain. We herein present a case of a patient with rAAA, and delayed abdominal closure was performed to prevent ACS.

\section{Case Report}

An 83-year-old male presented with sudden, severe abdominal pain. He underwent computed tomography (CT) at a neighboring hospital. The CT scan showed an $80 \times 85 \mathrm{~mm}$ infrarenal AAA with a large retroperitoneal hematoma (Fig. 1). He was brought to our hospital. Before his arrival, cardiac arrest occurred, and he received cardiopulmonary resuscitation. On admission, 


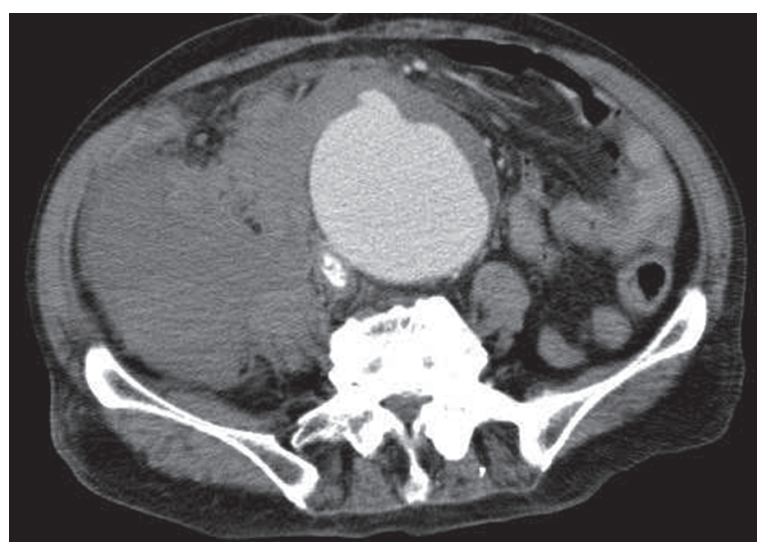

Fig. 1 Contrast-enhanced computed tomography (CT) scan showing an $80 \times 85 \mathrm{~mm}$ infrarenal AAA with a large retroperitoneal hematoma.

he presented with hemorrhagic shock, a systolic blood systolic pressure of $70 \mathrm{~mm} \mathrm{Hg}$, and clouded sensorium. His laboratory data showed a decreased hemoglobin level of $6.7 \mathrm{~g} / \mathrm{dl}$ and hematocrit of $23.7 \%$. He underwent an emergency operation. Bloody ascites were recognized in the abdominal cavity. A saccular type of infrarenal AAA measuring $80 \mathrm{~mm}$ in diameter and a large hematoma were observed in the retroperitoneal cavity. A ruptured hole was observed on the right lateral side of the aneurysm. The aneurysm was resected and reconstructed with a prosthetic bifurcated graft. The abdominal wall was distended because of the retroperitoneal hematoma and severe edematous bowel change. A delayed abdominal closure by the use of Bard Composix mesh ${ }^{\circledR}$ was performed to prevent ACS and confirm the resolution of bowel ischemia (Fig. 2). The operation time was 321 minutes and the blood loss was $2000 \mathrm{ml}$. The patient received a transfusion of 12 units of packed red blood cells. His postoperative body weight was increased by $13 \mathrm{~kg}$ over his preoperative weight. The patient remained intubated postoperatively. The postoperative daily drainage volume of the drain placed at the rectovesical pouch was $210 \mathrm{ml}$ on postoperative day (POD) $1,130 \mathrm{ml}$ on POD 2, $70 \mathrm{ml}$ on POD 3, and $70 \mathrm{ml}$ on POD 4. The postoperative daily drainage volume of the drain placed over the mesh side was $840 \mathrm{ml}$ on POD 1, $450 \mathrm{ml}$ on POD 2, $350 \mathrm{ml}$ on POD 3, and $265 \mathrm{ml}$ at POD 4. His body weight and abdominal circumference thereafter gradually decreased. The abdominal wall softened and the bowel sound was audible by 4 days after the primary operation, and at that time a secondary abdominal closure was performed. The mesh did not adhere

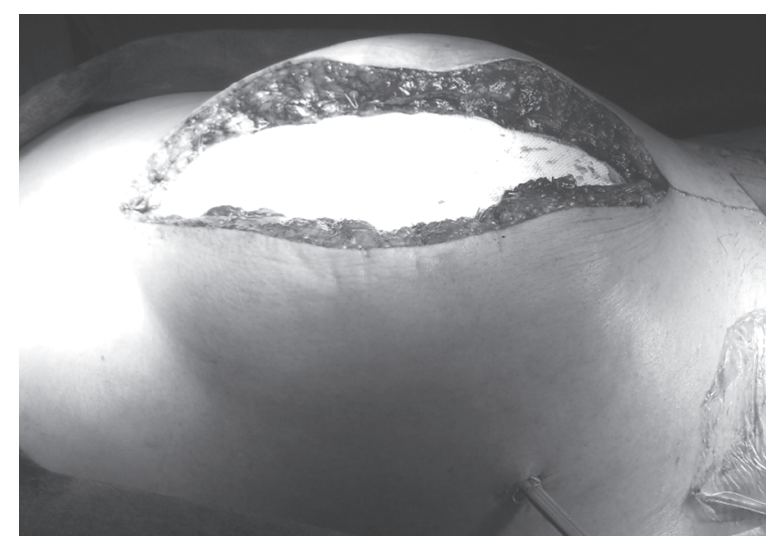

Fig. 2 The enteric canal was covered with Bard Composix mesh $^{\circledR}$, and the mesh side was fixed to the edge of the fascia.

to the viscera or peritoneum. As a result, the mesh was removed and the abdominal wall was closed easily. He was extubated soon after the secondary operation. Enteral nutrition was started by tube feeding 2 days after the secondary operation, and he could ingest by day 7 . He was discharged 21 days after the primary operation without intra-abdominal abscess, wound infection or incisional hernia.

\section{Delayed abdominal closure technique}

Firstly, a 19 French J-VAC silicone drain was placed at the rectovesical pouch to drain the fluid in the peritoneal cavity.

Secondly, Bard Composix mesh ${ }^{\circledR}$ (Bard, USA) was placed over the omentum and the enteric canal beneath the abdominal wall. Bard Composix mesh ${ }^{\circledR}$ is made of polyproplylene mesh on the upper side, bonded to an expanded polytetrafluoroethylene (ePTFE) sheet on the lower side. The mesh side was sutured to the fascial edges with interrupted sutures with bites of about $15 \mathrm{~mm}$. The ePTFE sheet does not adhere to the enteric canal or parietal peritoneum. Its interposition between the viscera and peritoneum prevents adhesion formation.

Thirdly, a 19 French J-VAC silicone drain was placed over the mesh side and tunnelled under the skin to exit $3 \mathrm{~cm}$ away from the wound edges.

Finally, the wound was covered with an isodine drape to keep the wound clean. Two J-VAC silicone drains placed at the rectovesical pouch and the wound were connected with $300 \mathrm{ml}$ closed drainage reservoirs, individually. This allowed drainage of the 


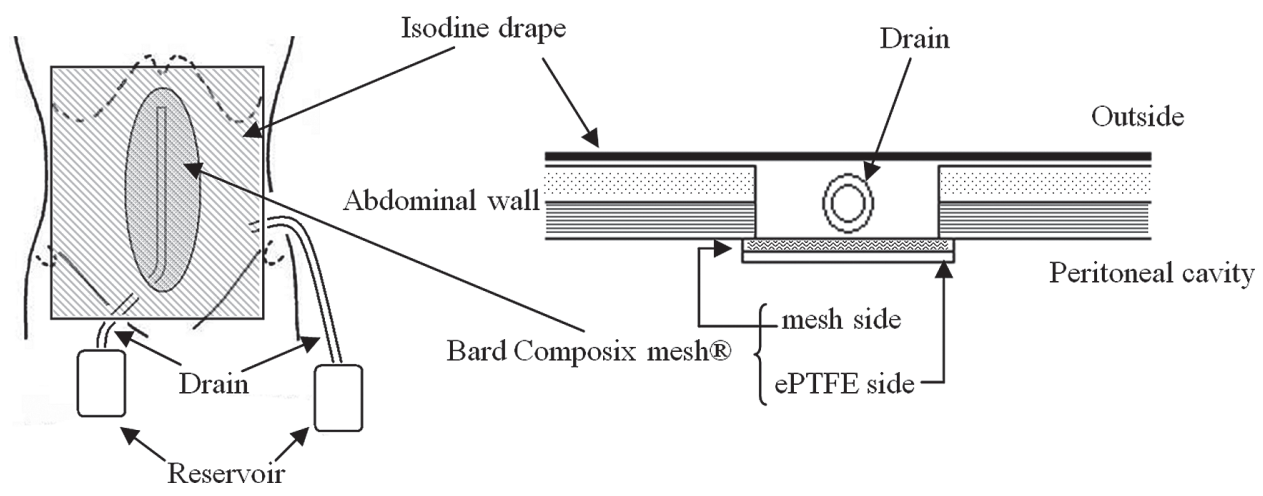

Fig. 3 Schematic presentation of the technique.

peritoneal cavity and the wound by continuous suction (Fig. 3).

\section{Discussion}

In patients surviving repair of rAAA, massive fluid resuscitation and blood transfusion predictably produce substantial visceral and abdominal wall edema. Additionally, these patients have typically had a period of tissue ischemia. Postoperative resuscitation in the setting of reperfusion injury leads to a systemic inflammatory response and secondary visceral edema., ${ }^{5,6)}$ Furthermore, the retroperitoneal hematoma surrounding an rAAA may displace the viscera anteriorly. In turn, visceral edema and retroperitoneal hematoma may lead to ACS. The prevalence of ACS after rAAA ranges from $10 \%-30 \%{ }^{7}$ ) The mortality rate after rAAA remains high because of the possibility of MOF in patients who survive the surgical repair. ACS is one of the causes of MOF after rAAA. ${ }^{3,4)}$ It is important to prevent ACS in order to improve the outcome of rAAA. A delayed abdominal closure is effective in preventing ACS in patients with rAAA. Rasmussen and colleagues proposed a delayed closure for patients with risk factors such as preoperative anemia, prolonged shock, and the need for cardiopulmonary resuscitation, intraoperative massive fluid resuscitation, severe acidosis, and profound hypothermia. ${ }^{8)}$

We successfully carried out delayed abdominal closure using Bard Composix mesh ${ }^{\circledR}$. Bard Composix mesh $^{\circledR}$ is the ventral hernia repair prosthesis that combines two clinically proven biomaterials, polypropylene mesh and ePTFE, in one product. It covers the abdominal wall easily and quickly without increasing the intra-abdominal pressure. The placement of J-VAC drains in the peritoneal cavity and on the mesh allowed for the effective drainage of any intra-abdominal fluid. It was important that one drain was placed in the peritoneal cavity and the other drain was placed outside the peritoneal cavity. Only the placement of a drain in the peritoneal cavity cannot allow for sufficient drainage. The intra-abdominal fluid may leak from the gaps between the abdominal wall and Bard Composix mesh ${ }^{\circledR}$ and accumulate in the isodine drape. As a result, intra-abdominal pressure cannot be decreased. Moreover, it is necessary to change the dressing or the risk of wound infection would increase. The addition of placement of a drain over the mesh side could the complete drainage. Postoperative wound management was straightforward because the abdominal wall was covered with an isodine drape and there was no need to change the dressing. The enteric canal was covered by the ePTFE side, and there was no risk of adhesion between enteric canal and abdominal wall. Moreover, ePTFE did not adhere to the enteric canal or parietal peritoneum. The mesh side was fixed to the edge of the fascia, and it was easy to remove. It produced a delayed abdominal closure with few adhesions, and the margin of the fascial seam was well preserved. If there is insufficient peritoneum or fascia, Bard Composix mesh ${ }^{\circledR}$ can remain in the abdominal wall, and a delayed closure can be performed only with skin closures.

Our delayed abdominal closure could be performed during emergency situations in a straightforward and rapid manner, and it was also easy to manage the wound. No infection was seen, the secondary closure could also be easily performed, and wound healing was good. Therefore, this delayed abdominal closure technique is considered to be useful for the treatment of rAAA. 


\section{Conclusion}

In an elderly male patient with an rAAA and hemorrhagic shock, we were able to successfully carry out surgical repair and delayed abdominal closure. Our delayed abdominal closure using the ventral hernia repair prosthesis could be performed in emergency situations in a straightforward and rapid manner, and it was also easy to manage the wound. This delayed abdominal closure technique is considered to be useful for the treatment of rAAA.

\section{Disclosure Statement}

The author declares that he has no conflict of interest.

\section{References}

1) Bown MJ, Sutton AJ, Bell PR, et al. A meta-analysis of 50 years of ruptured abdominal aortic aneurysm repair. Br J Surg 2002; 89: 714-30.

2) Noel AA, Gloviczki P, Cherry KJ, et al. Ruptured abdominal aortic aneurysms: the excessive mortality rate of conventional repair. J Vasc Surg 2001; 34: 41-6.
3) Bown MJ, Cooper NJ, Sutton AJ, et al. The postoperative mortality of ruptured abdominal aortic aneurysm repair. Eur J Vasc Endovasc Surg 2004; 27: 65-74.

4) Malbrain ML, Chiumello D, Pelosi P, et al. Incidence and prognosis of intraabdominal hypertension in a mixed population of critically ill patients: a multiplecenter epidemiological study. Crit Care Med 2005; 33: 315-22.

5) Oelschlager BK, Boyle EM, Johansen K, et al. Delayed abdominal closure in the management of ruptured abdominal aortic aneurysms. Am J Surg 1997; 173: 411-5.

6) Zakaria el R, Tsakadze NL, Garrison RN. Hypertonic saline resuscitation improves intestinal microcirculation in a rat model of hemorrhagic shock. Surgery 2006; 140: 579-87; discussion 587-8.

7) Bown MJ, Nicholson ML, Bell PR, et al. Cytokines and inflammatory pathways in the pathogenesis of multiple organ failure following abdominal aortic aneurysm repair. Eur J Vasc Endovasc Surg 2001; 22: 485-95.

8) Rasmussen TE, Hallett JW, Noel AA, et al. Early abdominal closure with mesh reduces multiple organ failure after ruptured abdominal aortic aneurysm repair: guidelines from a 10-year case-control study. J Vasc Surg 2002; 35: 246-53. 